\title{
Simulação do fator de atrito para o escoamento confinado de caulim com diferentes teores de sólidos pelo modelo de SWAMEE - JAIN
}

\section{Simulation of the friction factor for the confined flow of kaolin with different solid contents using the SWAMEE - JAIN model}

André Luis Mileo Ferraioli Silva ${ }^{1}$, André Luiz Amarante Mesquita ${ }^{2}$, Augusta Maria Paulain Ferreira Felipe ${ }^{1}$, José Antônio da Silva Souza ${ }^{1}$

\author{
${ }^{1}$ UFPA/ITEC/Departamento de Engenharia Química CEP: 66075-110, Belém - PA \\ e-mail: andremileo@ufpa.br, ampf@ufpa.br,jass@ufpa.br \\ ${ }^{2}$ UFPA/ITEC/Departamento de Engenharia Mecânica \\ e-mail: andream@ufpa.br
}

\section{RESUMO}

Estimar perdas de carga devido à fricção em tubos fechados é uma tarefa importante na solução de muitos problemas práticos nos diferentes ramos da profissão de engenharia. O projeto hidráulico e análise de sistemas de escoamento de suspensão mineral são dois exemplos. Muitos consideram o modelo de Swamee - Jain promissor para avaliar as perdas de carga devido ao atrito em conduto de tubos fechados. Para investigar o fator de atrito o modelo de Swamee - Jain foi empregado para o escoamento confinado de suspensão de caulim com teor de sólidos em massa de 55\% (suspensão C), 60\% (suspensão B) e 65\% (suspensão A) em tubulação de ferro galvanizado com 0,2; 0,3 e 0,5 metros de diâmetro com faixa de vazão de 0,02 a $0,2 \mathrm{~m}^{3}$ por segundo.Todas as suspensões de caulim o aumento do teor de solido e do diâmetro da tubulação contribui para o aumento do fator de atrito para o escoamento confinado em faixa de vazão operacional proporcionando estimativa de valores para utilização em projeto e analise de sistemas de escoamento de caulim.

Palavras-chave: caulim, fator de atrito, reologia, modelo de Swamee - Jain.

\begin{abstract}
Estimating load losses due to friction in closed pipes is an important task for solving many practical problems in the various branches of the engineering profession. Hydraulic projects and analyses of systems for transporting mineral products in suspension are two examples. Many consider the Swamee Jain model a promising method for assesses load losses due to friction in closed pipes. In order to investigate the friction factor the Swamee - Jain model was employed for confined flow of a kaolin suspension with a concentration of solids in mass of 55\% (suspension C), $60 \%$ (suspension B) and $65 \%$ (suspension A) in a galvanized iron pipe with $0.2 ; 0.3$ and 0.5 meters in diameter with a flow ranging from 0.02 to $0.2 \mathrm{~m}^{3}$ per second. For all of the kaolin suspensions the increase in the solid content and the diameter of the pipe contributes towards increasing the friction factor for confined flow in the operational flow, providing an estimate of values for use in projects and for analyzing systems used for transporting kaolin.
\end{abstract}

Keywords: kaolin, friction factor, rheology, Swamee - Jain model.

\section{INTRODUÇÃO}

O escoamento eficiente de suspensão de Caulim depende da otimização do projeto hidráulico, buscando sempre a menor soma dos custos fixos e variáveis. Neste caso, um dos parâmetros mais importantes é a perda de carga, a qual deve ser determinada com precisão, resultando no sistema de recalque mais econômico. 
O modelo Swamee - Jain (1976), citados por Porto [9], apresentaram uma expressão geral dada pela equação (1) que calcula o fator de atrito (f) para faixa de rugosidade relativa de $1.10^{-6}$ a 0,001 e para numero de Reynold inferior a $1.10^{8}[5]$. O modelode Swamee - Jain é empregado para estimar o fator de atrito em regime turbulento apresentando boa convergência também para o regime laminar [15],[1]. Tem a vantagem de se aplicar a qualquer regime de escoamento, o que simplifica a formulação dos problemas nos quais não se conhece a ordem de grandeza do número de Reynolds [6].

$f=\left\{\left(\frac{64}{R e}\right)^{8}+9,5\left[\operatorname{Ln}\left(\frac{1}{3,7} \cdot \frac{\varepsilon}{D}+\frac{5,74}{R e^{0,9}}\right)-\left(\frac{2500}{R e}\right)^{6}\right]^{-16}\right\}^{0,125}$

O modelo de Swamee-Jain é de aplicação geral e recentemente já vem sendo implementado pelo setor mineral como alternativa aos modelos clássicos. Com a validação do modelo de Swamee-Jain o setor mineral requer e incentiva sua aplicação e avaliação no processo de escoamento de diferentes suspensões minerais.

Os caulins são constituídos por argilo-minerais do grupo da caulinita, são eles a própria caulinita, a haloisita e metahaloisita, a nacrita e a diquita. Embora esses minerais tenham composição química similar, cada um deles possui importantes diferenças estruturais [7], [10], [12].

As suspensões aquosas de caulim são constituídas de partículas de ordem coloidal e com elevadas concentrações de sólidos. As propriedades reológicas de sistemas coloidais dependem da viscosidade do líquido dispersante, da concentração da fase dispersa, do tamanho e forma das partículas dispersas e das forças de interação, ou seja, do estado de estabilidade da suspensão [8].

$\mathrm{O}$ beneficiamento do minério de caulim é realizado através de via úmida sob a forma de suspensão, que necessita ser transportada, bombeada, ao longo do processo [13], [3]. Daí a importância do fator de atrito para o correto dimensionamento do sistema de bombeamento de forma a evitar o super dimensionamento e consumo desnecessário de energia [14].

\section{MATERIAIS E MÉTODOS}

\subsection{Caracterização química da matéria-prima}

O caulim da suspensão aquosa, de origem da formação barreiras a margem do rio capim município de ipixuna do Pará, foi submetido à caracterização química através da técnica de fluorescência de raios-X utilizando espectrômetro WDS sequencial, modelo Axios Minerals PANalytical, com tubo de raios-X cerâmico e anodo de ródio.

\subsection{Característica da suspensão de Caulim}

As suspensões de caulim a ser determinado o fator de atrito pelos modelos apresentam um teor de sólidos de $65 \%$ (suspensão A), $60 \%$ (suspensão B) e 55\% (suspensão C) em massa. As suspensões A, B e C possuem massa especifica de $1510 \mathrm{~kg} / \mathrm{m}^{3}, 1473 \mathrm{~kg} / \mathrm{m}^{3}$ e $1412 \mathrm{~kg} / \mathrm{m}^{3}$ respectivamente. O caulim possui um diâmetro médio de partículas no valor de $83 \mu \mathrm{m}$, e o pH das suspensões aquosa de caulim é de 5,3. Essas são condições operacionais em operação de bombeamento.

\subsection{Reologia}

A suspensão de caulim foi ensaiada em viscosímetro VT 550 modelo Haake com cilindros coaxiais SV1 (spindle) a $25{ }^{\circ} \mathrm{C}$ para obtenção de curva experimental de viscosidade aparente em função da taxa cisalhante de 0 a $700 \mathrm{~s}^{-1}$ com seus respectivos intervalos de erro, através da programação reologia em rampa de aumento de taxa cisalhante de 0 a $700 \mathrm{~s}^{-1}$ com duração total de $120 \mathrm{~s}$. Após a obtenção da curva experimental foi ajustado o modelo matemático de Hershel Bulkley o que possibilita a estimativa do fator de atrito pelo modelo de Swamee - Jain para as condições operacionais de escoamento. 


\subsection{Condições operacionais de simulação de escoamento}

Para utilização do modelo de Swamee - Jain se adotou as especificações estruturais e operacionais para o sistema de bombeamento apresentado pela tabela 1 .

Tabela 1: Condições Operacionais de escoamento

\begin{tabular}{c|c}
\hline Tubulação: & Ferro Galvanizado com rugosidade $0,15 \mathrm{~mm}$ \\
Faixa de Diâmetro: & 0,$2 ; 0,3$ e 0,5 metros \\
Faixa de Vazão: & 0,02 a $0,2 \mathrm{~m}^{3}$ por segundo \\
Faixa de Numero de Reynolds & 244,8 a 175068,1 \\
\hline
\end{tabular}

Pela tabela 1 o material da tubulação é ferro galvanizado e para ensaio do modelo se tem que para cada um dos três diâmetros hidráulicos da tubulação cilíndrica se avaliou escoamento do caulim numa faixa de vazão de 0,02 a $0,2 \mathrm{~m}^{3}$ por segundo. A rugosidade de $0,15 \mathrm{~mm}$ engloba o ferro galvanizado $[2]$.

\section{RESULTADOS E DISCUSSÃO}

\subsection{Composição química das matérias-primas}

A Tabela 2 apresenta a composição química do caulim da suspensão ensaiada.

Tabela 2: Analise Química do Caulim

\begin{tabular}{|c|c|c|c|c|c|c|c|}
\hline & $\mathrm{SiO}_{2}$ & $\mathrm{Al}_{2} \mathrm{O}_{3}$ & $\mathrm{Fe}_{2} \mathrm{O}_{3}$ & $\mathrm{Na}_{2} \mathrm{O}$ & $\mathrm{P}_{2} \mathrm{O}_{5}$ & $\mathrm{TiO}_{2}$ & P. $F^{*}$ \\
\hline CAULIM & 46,64 & 37,75 & 0,53 & 0,23 & 0,11 & 0,39 & 14,33 \\
\hline
\end{tabular}

Pela Tabela 2 observa-se que o caulim de origem da formação barreiras a margem do rio capim, apresenta em sua composição $\mathrm{SiO}_{2}(46,64 \%)$. O resultado da fluorescência mostrou ainda um teor considerável de $\mathrm{Al}_{2} \mathrm{O}_{3}(37,75 \%)$ característico do caulim.

\subsection{Reologia}

A figura 1 apresenta a curva experimental da viscosidade aparente versus taxa cisalhante para a suspensões $\mathrm{A}(65 \%), \mathrm{B}(60 \%)$ e $\mathrm{C}(55 \%)$ de caulim com seus respectivos intervalos de erro e apresenta também o ajuste do modelo Herschel-Bulkley para os dados experimentais. 


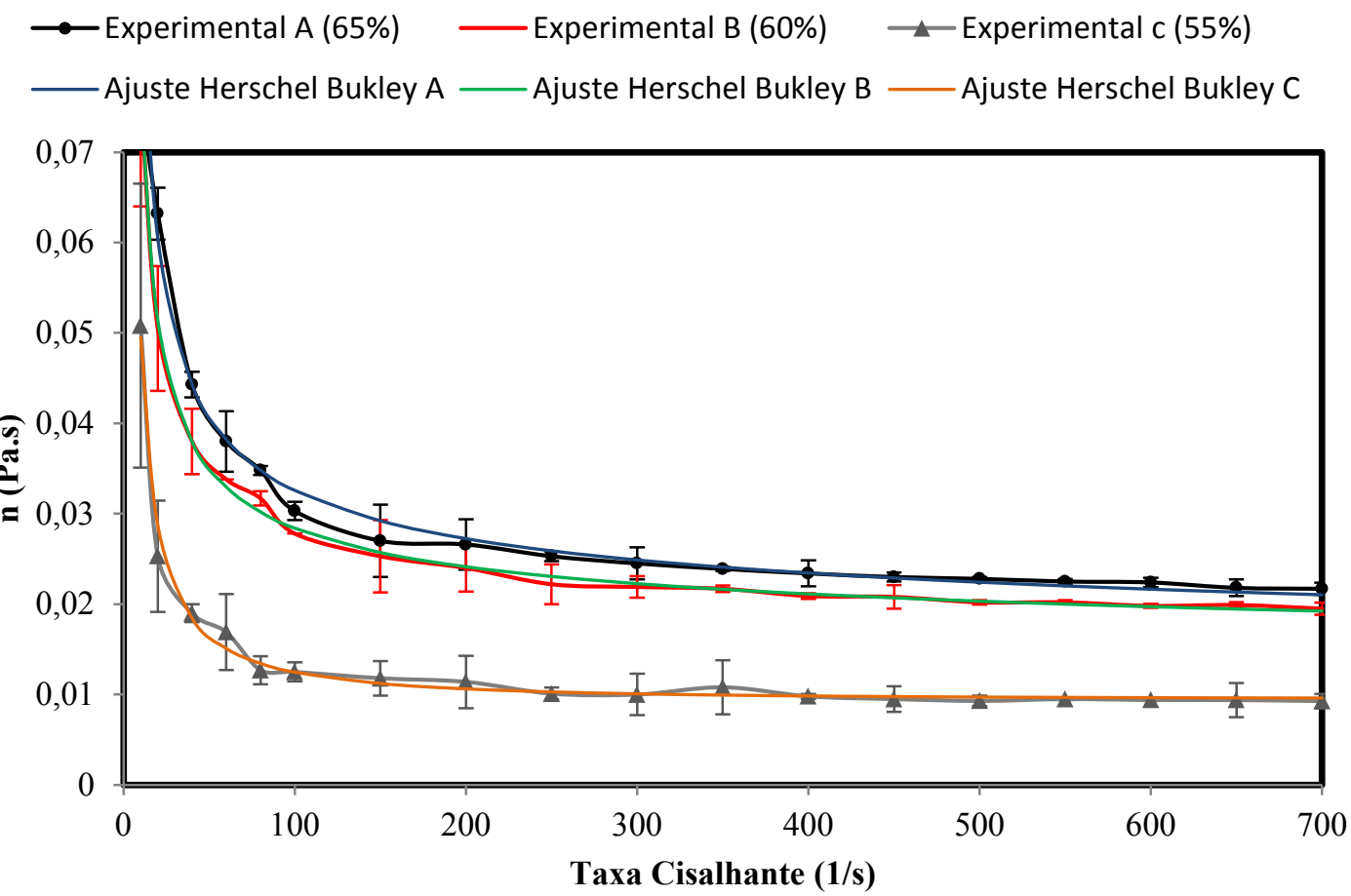

Figura 1: Curva Experimental de viscosidade versus taxa cisalhante com ajuste de modelo Herschel-Bulkley.

Pela figura 1 observa-se que as suspensões de caulim A, B e C apresentam comportamento não newtoniano, pois a viscosidade decresce com o aumento da taxa cisalhante. A suspensão " A' por ter maior teor de sólidos $65 \%$ apresentou maior viscosidade que as suspensões " $B$ " e "C" o que é constatado pela curva superior na figura 1 .

De acordo com a figura 1 observa-se o ajuste do modelo Herschel-Bulkley dado pelas equações (2), (3) e (4) para as suspensões de caulim $\mathrm{A}(65 \%), \mathrm{B}(60 \%)$ e $\mathrm{C}(55 \%)$ respectivamente, sendo representado pela curva sem intervalo de erro. Esse modelo apresentou bom ajuste aos valores experimentais com um coeficiente de correlação de 0,979 para a suspensão A, 0,998 para o B e de 0,994 para o C.

$\eta=\frac{0,4978}{\gamma}+0,057 \gamma^{0,8426-1}$

$\eta=\frac{0,4315}{\gamma}+0,0443 \gamma^{0,8675-1}$

$\eta=\frac{0,4204}{\gamma}+0,006754 \gamma^{1,044-1}$

Pelas equações (2), (3) e (4) é possível relacionar viscosidade com taxa cisalhante, sendo que essa relação é útil para aplicação do modelo de Swamee - Jain para estimativa do fator de atrito para as condições operacionais.

A viscosidade depende da taxa de cisalhamento pelo fato da suspensão de caulim ser um fluido não newtoniano [11], [4], e a taxa de cisalhamento é função da vazão e do diâmetro da tubulação.

A figura 2 apresenta os resultados da viscosidade aparente $(\eta)$ em função da vazão volumétrica para a suspensão de caulim escoando na tubulação de ferro galvanizado com os respectivos diâmetros. 




Figura 2: Curva de viscosidade versus vazão volumétrica

De acordo com a figura 2 para todos os diâmetros se tem a diminuição da viscosidade aparente com o aumento da vazão devida o aumento da velocidade de escoamento.

Para as tubulações de ferro galvanizado de diâmetro hidráulico 0,2;0,3 e 0,5 metros a partir de 80 litros por segundo se tem o estabelecimento do regime turbulento fazendo com que a viscosidade aparente se mantenha com baixo decréscimo numa faixa de 0,1187 a 0,0124 Pa.s.

A tubulação de 0,5 metros possui um decréscimo mais acentuado de viscosidade devido à menor velocidade de escoamento fazendo com que as forças viscosas prevaleçam em relação às forças inerciais ao longo do escoamento.

Pela figura 2 o aumento do teor de sólidos e do diâmetro da tubulação tende a aumentar o valor da viscosidade para faixas de escoamento operacional.

A figura 3 apresenta os resultados do fator de atrito obtido pelo modelo de Swamee - Jain. 


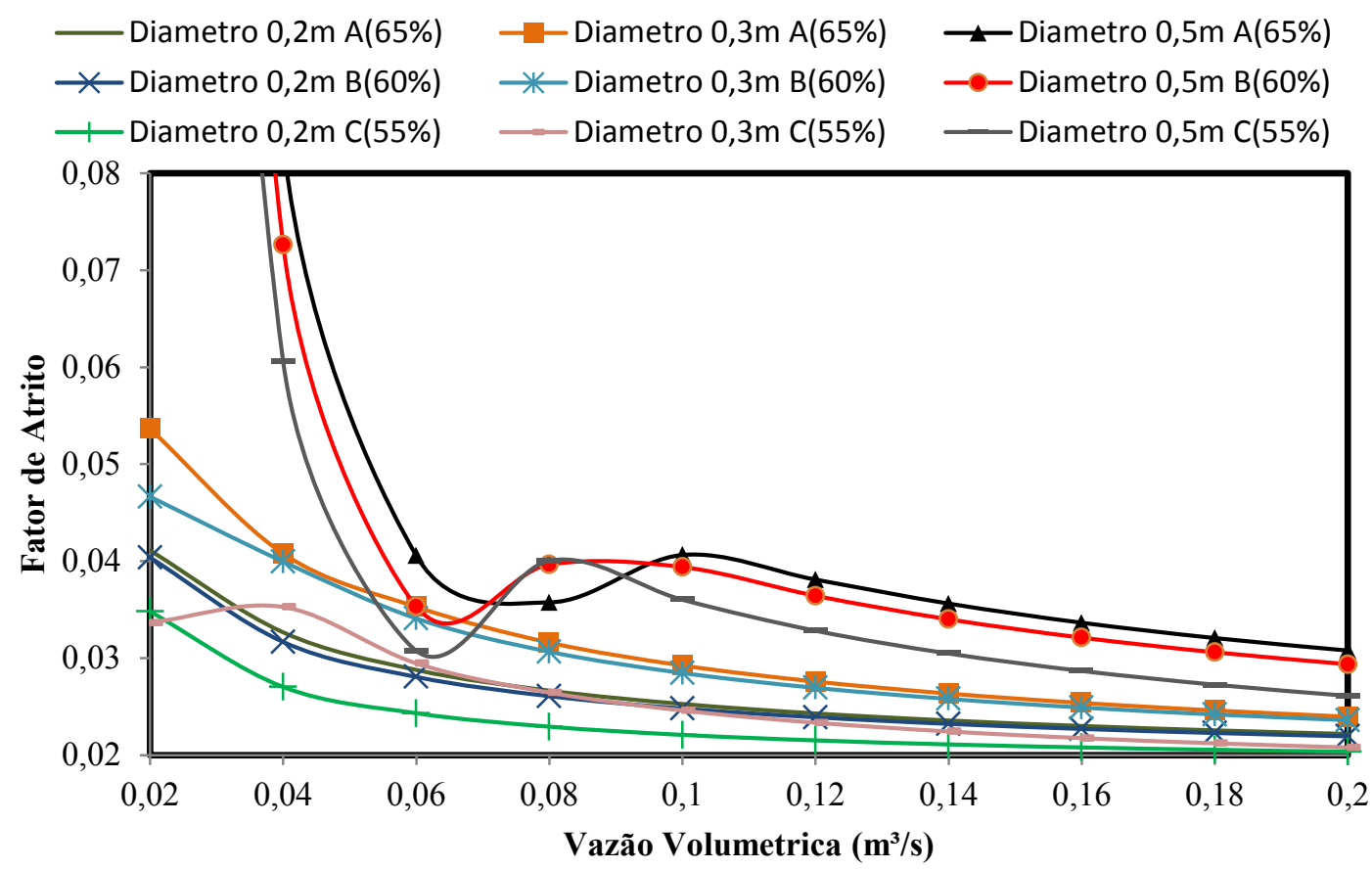

Figura 3: Fator de Atrito pelo modelo de Swamee - Jain

A figura 3 apresenta os resultados do fator de atrito estimado pelo modelo de Swamee - Jain, para todos os diâmetros em analise o fator diminui com o aumento da vazão devido o aumento do numero de Reynolds.

Para as tubulações de maior diâmetro, para uma mesma vazão, possui menor velocidade de escoamento que por sua vez menor Reynolds o que justifica o aumento do fator de atrito para os maiores diâmetros de tubulação.

Pela figura 3 se tem que o aumento do teor de sólidos contribui para o aumento do fator de atrito como se pode observa os valores de fator de atrito de 0,0416; 0,0399 e 0,0361 para as suspensões $\mathrm{A}(65 \%), \mathrm{B}(60 \%)$ e $\mathrm{C}(55 \%)$ respectivamente escoando em tubulação de ferro galvanizado de 0,5 metros de diâmetro a uma vazão de $0,1 \mathrm{~m}^{3} / \mathrm{s}$. O aumento do teor de sólidos acarreta no aumento de sílica livre na suspensão o que contribui para o aumento do fator de atrito.

As oscilações nas curvas da figura 3 para uma dada vazão é devido à transição de o regime laminar para o turbulento.

\section{CONCLUSÃO}

As suspensões de caulim $\mathrm{A}(65 \%), \mathrm{B}(60 \%)$ e $\mathrm{C}(55 \%)$ possuem boa operacionalidade nas tubulações de 0,2 e 0,3 metros de diâmetro para toda a faixa de vazão em analise de 0,02 a $0,2 \mathrm{~m}^{3}$ por segundo devido apresentarem valores moderados de fator de atrito e de velocidade de escoamento.

Para a tubulação de 0,5 metros a faixa de vazão de 0,08 a $0,2 \mathrm{~m}^{3}$ por segundo apresentou, boa operacionalidade para todas as suspensões devido aos valores moderados de fator de atrito e de velocidade de escoamento.

Pelo modelo de Swamee - Jain em rugosidade relativa de $1.10^{-6}$ a 0,001 e numero de Reynold inferior a $1.10^{8}$, o aumento do teor de solido e do diâmetro da tubulação para faixa operacional de vazão contribui para o aumento do fator de atrito no escoamento confinado de caulim em tubulação de ferro galvanizado possibilitando o projeto e o acompanhamento do escoamento de caulim de forma mais precisa, contribuindo para a diminuição do consumo energético no setor mineral. 


\section{BIBLIOGRAFIA}

[1] ANDRADE, L., CARVALHO, A. "Análise da equação de Swamee-Jain para cálculo do fator de atrito”, Revista Brasileira de Engenharia Agricola e Ambiental, v. 5. pp. 554-557, 2001.

[2] AZEVEDO, N.J.M., FERNANDEZ, F.M., ARAUJO, R., et al. Manual de hidráulica. 8.ed. São Paulo, Blücher, , 1998.

[3] BARATA, M.S., ANGÉLICA, R.S. "Atividade pozolânica dos resíduos cauliníticos das indústrias de mineração de caulim da Amazônia”, Revista Matéria. v.16, pp. 795-810, 2011.

[4] BURGER, J., HALDENWANG, R., ALDERMAN, N. "Friction factor-Reynolds number relationship for laminar flow of non-Newtonian fluids in open channels of different cross-sectional shapes". Chemical Engineering Science, v.65, p. 3549-3556, 2010.

[5] DURAND, A.I. A, SEGOVIA, G.I.C, BÁRCENAS, O.F, et al., "Evaluación de ecuaciones de factor de fricción explícito para tuberías", Educación Química, v.25. p 128-134, 2014

[6] GOMIDE, R., "Operações Unitárias - Volume II - Fluidos na Indústria”, 1 .ed, Reynaldo Gomide, pp 432, 1993.

[7] HU, Y., LIU, X. "Chemical Composition and Surface Properties of Kaolins", Minerals Engineering, v.16, n.11, p. 1279-1282, 2003.

[8] LUZ, A.B., CHAVES, A.P. Processo de Beneficiamento de Caulim com Estabilização dos Íons Ferrosos, Pedido de Patente PI 9803302. (2000).

[9] PORTO, R.M. Hidráulica básica. São Carlos, EESC-USP. 540p. 1998

[10] SANTOS, E., SCORZELLI, R.B., BERTOLINO, L.C., et al., "Characterization of kaolin from the Capim River region, Brazil”, Appl. Clay Sci. V.55, 164-167, 2012.

[11] SILVA, A.L.M.F., CARDOSO, D.N.P., FELIPE, A.M.P.F., et al. "Influência de aditivo PVA no comportamento reológico de argamassa elaborada a partir de resíduos Industriais", Cerâmica Industrial (Impresso), v. 20, p. 24-29, 2015.

[12] SOUZA, D.J.L., VARAJÃO, A.F.D.C., YVON, J., "Geochemical evolution of the Capim River kaolin, Northern Brazil"., J. Geochem. Explor. 88, 329-331, 2006.

[13] VARELA, J. J., GLIESE, R., PETTER, C. O., et al., "Controle de qualidade no processamento de polpas de caulim utilizando propriedades óticas", Rem: Revista Escola de Minas, v. 58, p. 201-206, 2005.

[14] VLASAK, P., CHARA, Z. "Laminar and turbulent flow experiments with yield power-law slurries". Powder Technology 104, pp. 200-206, 1999.

[15] WINNING, H.K., COOLE, T., "Explicit friction factor accuracy and computational efficiency for turbulent flow in pipes", Flow, Turbul. Combust, v. 90, pp. 1-27, 2013. 